\title{
Capillary Hemangioma of the Nasal Septum: A Rare Case Report
}

\author{
R. Farid ${ }^{1}$, Saim Aminuddin², E. A. Ihab ${ }^{3 *}$, Loh Keng-Yin ${ }^{4}$ \\ ${ }^{1}$ ENT Department, Hospital Sungai Buloh, Kuala Lumpur, Malaysia \\ ${ }^{2}$ KPJ AmpangPuteri, Hospital, Kuala Lumpur, Malaysia \\ ${ }^{3}$ ENT Department, School of Medicine, Taylors University, Selangor, Malaysia \\ ${ }^{4}$ Family Medicine Department, School of Medicine, Taylors University, Selangor, Malaysia \\ Email: ^ihab.ali@taylors.edu.my
}

How to cite this paper: Farid, R., Aminuddin, S., Ihab, E.A. and Keng-Yin, L. (2020) Capillary Hemangioma of the Nasal Septum: A Rare Case Report. International Journal of Otolaryngology and Head \& Neck Surgery, 9, 213-217.

https://doi.org/10.4236/ijohns.2020.96025

Received: September 10, 2020

Accepted: November 1, 2020

Published: November 4, 2020

Copyright (c) 2020 by author(s) and Scientific Research Publishing Inc. This work is licensed under the Creative Commons Attribution International License (CC BY 4.0).

http://creativecommons.org/licenses/by/4.0/

\begin{abstract}
Capillary hemangioma is a congenital malformation of the capillary. It commonly affects the face, eyelids, lips and skin. Adult capillary hemangioma involving the nasal cavity is rare and may be misdiagnosed as polyps or another tumor. This case report illustrated a middle-age adult patient in Malaysia who presents with unilateral nasal obstruction and intermittent epistaxis who later confirmed capillary hemangioma involving the right nasal septum. Endoscopic surgery excision of the hemangioma was successfully performed. There were no known risk factors present in him. A treating doctor in the outpatient clinic seeing symptoms such as unilateral nasal obstruction must consider alternative diagnosis such as hemangioma besides usual diagnosis of polyps.
\end{abstract}

\section{Keywords}

Capillary Hemangioma, Nasal, Septal, Bleeding

\section{Introduction}

Capillary hemangioma is also known as strawberry nevi, characterized by tiny reddish nodule on the body. It is a benign condition due to abnormal overgrowth of blood vessels [1]. Although the majority of the capillary hemangiomas present as single nodular lesion, multifocal disease had been reported in children [2]. This condition can affect many parts of the body surface and internal organ, while head and neck region is one of the most common affected sides. Among the structure affected are face, eye lids, gingiva, oral mucosa, tongue and lips [3]. Capillary hemangioma affecting the nasal or sinus cavity is rare, accounting for 
only about $10 \%$ of all head and neck region hemangioma [4]. The pathogenesis of this lesion is belief to be related to hormonal factors when the fetus is in utero [5]. When the lesions involve the nasal mucosa, the common presenting symptoms are nasal obstruction and epistaxis. The following case report illustrated a 46-year-old patient in Malaysia presented with nasal obstruction due to a nasal mass which subsequently confirmed a capillary hemangioma.

\section{Case History}

A 46-year-old man presented with one-month history of left nasal obstruction with intermittent epistaxis. There was no history of trauma or infection prior to these symptoms. There was no significant past history of medical disorder such as bleeding disorder nor any significant childhood history of illnesses. Besides the intermittent left nasal obstruction and intermittent epistaxis, he was totally well and able to carry out his daily activity. There was no history of fever, headache, loss of weight nor loss of appetite. His smell, hearing and swallowing were normal Inspection of the nasal cavity reveals an erythematous polypoid mass covering the entire left nasal cavity. Part of the soft tissue mass is protruding out from the nostril (Figure 1). There was contact bleeding when the mass was touched. The right nasal cavity is normal. Examination of the ear and oral cavity were normal. There was no palpable cervical lymphadenopathy. A nasal endoscopy was performed, and it was noted the nasal mass was connected to the anterior part of left nasal septum via a stalk. Further investigation by CT scan of nasal and paranasal sinuses revealed a soft tissue mass arising from the anterior superior portion of the left nasal septum, without connection to the intracranial contents. The mass was seen occupied 50 percent of the left nasal cavity (Figure 2). A preliminary diagnosis of a benign nasal soft tissue tumor was made. In view of the obstructive nature of the mass it was decided excision of the nasal mass was necessary. The patient consented to the surgery and proceeded to endoscopic surgery under general anesthesia. His hemoglobin level, full blood count, kidney and liver functions results were all within normal range. The procedure

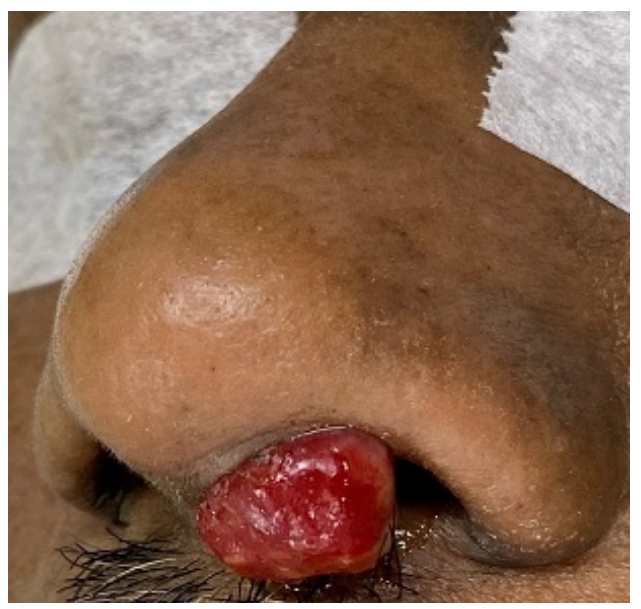

Figure 1. A soft tissue mass protruding from the left nostril. 
was successfully completed without any complications. The entire hemangioma was completely excised at the base with the intact stalk (Figure 3). There was minimal bleeding without the need of preoperative embolization or perioperative blood transfusion. The excised mass was sent for histopathological examination (HPE). The final HPE reported the histology findings of the mass consistent with a capillary hemangioma.

\section{Progress and Outcome}

Following surgery, the patient recovered well without any complication. He was discharged from hospital after five days of hospitalization. Four weeks later he was followed up again at the ENT clinic and his symptoms of epistaxis and nasal obstruction were totally subsided. Examination of the nasal cavity did not show any local complications such as edema, deformity or bleeding. The nostril airway appears patent. Subsequent follow up of the patient revealed no evidence of residual or recurrent. Till now he remains asymptomatic and healthy.

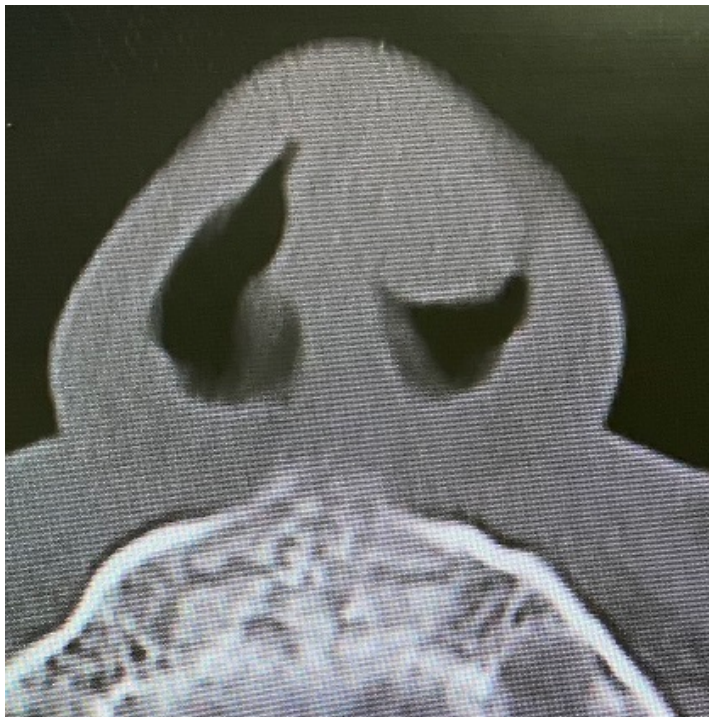

Figure 2. More than half of the left nasal cavity was occupied by the hemangioma.

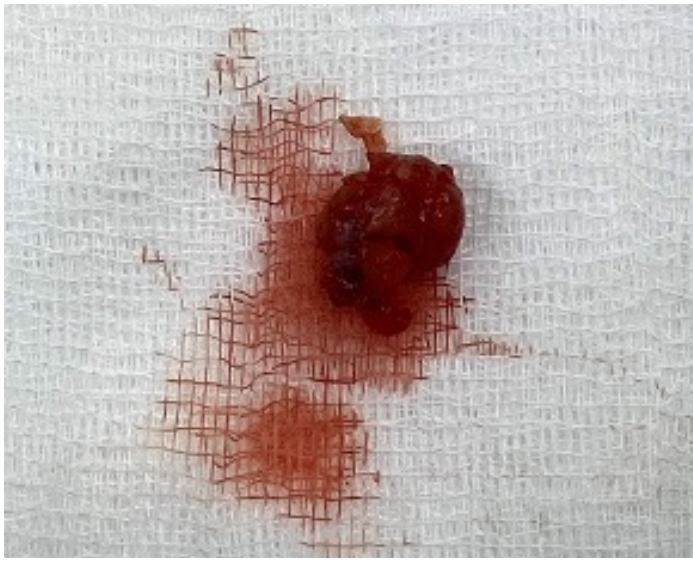

Figure 3. Hemangioma removed completely via endoscopic nasal surgery. 


\section{Discussion}

Capillary hemangioma is a congenital malformation of blood vessels, affecting mostly children. However, it may not manifest in early childhood until early adulthood and the symptoms only present in early adulthood due the slow growing nature of the lesion. This condition is rare in adulthood after the age of 40. [1] The patient presented in this case was a middle age adult, there were not many published reports of capillary hemangioma at this age. The other epidemiological factor worth mentioned is middle adulthood female has preponderance to male in the prevalence of nasal hemangioma but not in this case.

The other diagnostic challenge illustrated in this case was the presenting features. The patient presents with unilateral nasal obstruction with intermittent epistaxis. This is a common presenting feature of nasal polyps. However, this patient did not have other features commonly seen in nasal polyps such or foul-smelling discharge. This makes the diagnosis of nasal polyps less likely, therefore the treating doctor must think of alternative diagnosis. On examination, the contact bleeding that occurred makes the diagnosis of capillary hemangioma more likely. Other symptoms commonly reported associated with nasal hemangioma besides epistaxis are headache, facial pain or mucopurulent discharge were not present [6].

The etiology of the capillary hemangioma is undetermined in this patient. There was no known past history of any childhood diseases, trauma or family history of similar condition in the family.

In terms of treatment, one of the most important factors to be considered is hemodynamic of the patient. Anemia due to chronic bleeding is one of the known complications. This patient did not have the problem, thus allowed him to proceed to endoscopic surgical removal of the mass which was completed successfully. He also did not require more complex procedures such as pre-operative embolization.

\section{Conclusion}

Capillary hemangioma of nasal septum is a rare lesion in middle adult man which can occur without any known etiology or cause. This case illustrated unilateral nasal obstruction and contact bleeding seen on a soft tissue mass in the nasal cavity must alert the treating doctor the possibility of a hemangioma besides the usual diagnosis of nasal polyp. Endoscopic excision without pre-operative embolization, usually appropriate in patients without complications such as anemia or massive bleeding, the recovery and prognosis, is good.

\section{Conflicts of Interest}

The authors declare no conflicts of interest regarding the publication of this paper.

\section{References}

[1] (2017) Capillary Hemangioma. American Association for Pediatric Ophthalmology 
and Strabismus. https://aapos.org/glossary/capillary-hemangioma

[2] Nedev, P. (2008) Lobular Capillary Haemangioma of the Nasal Cavity in Children. Trakia Journal of Sciences, 6, 63-67.

[3] Genc, S., Kurkcuoglu, S.S., Karabulut, H., Acar, B., Tuncel, U. and Degerli, S. (2009) Giant Lobular Capillary Hemangioma of the Nasal Septum. Turkish Journal of Medical Sciences, 39, 325-328.

[4] Kim, S.T., Kim, S.H., Gu, G.Y. and Cha, H.E. (2000) Three Cases of Hemangioma in Nasal Septum. Journal of Rhinology, 7, 80-83.

[5] Nair, C.S., Bahal, M.A. and Bhadauria, R.S. (2008) Lobular Capillary Hemangioma of Nasal Cavity. Medical Journal Armed Forces India, 64, 270-271. https://doi.org/10.1016/S0377-1237(08)80114-1

[6] Capelli, M. and Patrizia, G. (2015) A Case of Capillary Hemangioma of the Nasal Septum in a Patient with Chronic Ischemic Heart Disease and Antiplatelet Treatment Non-Suspendable. Journal of Medical Cases, 6, 59-61. https://doi.org/10.14740/jmc2022w https://www.journalmc.org/index.php/JMC/article/view/2022/1418 